\title{
Bayesian Genetic Programming for Edge Detection
}

\author{
Wenlong Fu · Mengjie Zhang · Mark Johnston
}

Received: date / Accepted: date

\begin{abstract}
In edge detection, designing new techniques to combine local features is expected to improve detection performance. However, how to effectively design combination techniques remains an open issue. In this study, an automatic design approach is proposed to combine local edge features using Bayesian programs (models) evolved by Genetic Programming (GP). Multivariate density is used to estimate prior probabilities for edge points and non-edge points. Bayesian programs evolved by GP are used to construct composite features after estimating the relevant multivariate density. The results show that GP has the ability to effectively evolve Bayesian programs. These evolved programs have higher detection accuracy than the combination of local features by directly using the multivariate density (of these local features) in a simple Bayesian model. From evolved Bayesian programs, the proposed GP system has potential to effectively select features to construct Bayesian programs for performance improvement.
\end{abstract}

Keywords Genetic Programming · Edge Detection . Bayesian Model · Feature Construction

\section{Introduction}

In order to extract information from an object, an essential job is to distinguish the object from its back-

Wenlong Fu. and Mengjie Zhang

School of Engineering and Computer Science, Victoria University of Wellington, PO Box 600, Wellington, New Zealand

Tel.: +64-4-4635233-7059

Fax: +64-4-4635045

E-mail: Wenlong.Fu@gmail.com

Mark Johnston

University of Worcester, United Kingdom. ground. Edge detection is a process of detecting discontinuous changes between objects and background in an image [2, 37]. In general, the edges extracted from an image can be simplified to describe the image content. Edge detection benefits a wide range of applications, such as image compression [33], object tracking [35], and image retrieval [23]. In a grayscale image, an edge feature for each pixel is a general mathematical formula of intensity values of pixels in a local area, and it is employed in the process of marking the pixel as an edge point or a non-edge point. To extract edge features, many methods have been proposed [30, 15, 3]. From human observations, several different solutions might be considered as true edge maps of one natural image. Therefore, edge detection is a subjective task. Different local edge features have been combined to improve detection performance $[28,9]$.

Our previous work [11] has shown that Genetic Programming (GP) can be successfully applied to feature construction by combining basic features and estimating the distribution of the observations of evolved programs. The basic edge features come from different prior domain knowledge (the Gaussian gradient, the normalised standard deviation, and the histogram gradient). The constructed GP edge features were significantly better than the combination from a simple Bayesian model using a general multivariate normal density [7].

From machine learning [7], different methods, such as Bayesian techniques, can be applied for combining basic features. Bayesian techniques have been widely applied to classification [21]. Since these techniques are based on applying Bayes' theorem [7], human experts can understand the models using the Bayesian techniques. However, there are still existing issues in the Bayesian techniques, such as how to effectively select features and design Bayesian models [21]. It is there- 
fore desirable to develop a GP system to automatically select features and design Bayesian models for performance improvement of a Bayesian technique.

The existing work using GP in edge detection mainly focuses on the construction of low-level edge detectors, such as approximating one-dimen-sional filters [31], selecting raw pixels [38], and combining image operators [22]. The work using GP in edge detection for the construction of low-level edge detectors shows that GP has potential to effectively evolve good edge detectors $[38,22,10]$. We have conducted initial investigation of employing GP to evolve Bayesian programs [14]. The evolved programs have higher detection accuracy than a simple Bayesian model to combine a set of predefined basic edge features.

\subsection{Goal}

The goal of this paper is to investigate automatic highlevel feature construction for edge detection using GP to evolve Bayesian programs. Here, a high-level feature means that it is constructed based on some basic features and combination techniques with prior domain knowledge. In our proposed Bayesian GP system [14], a simple Bayesian model with a general multivariate normal density was proposed as a function to construct different Bayesian programs. Different from our previous work [14], this paper further investigate the Bayesian GP system. The simple Bayesian model can be used as a terminal as well. How to effectively use the Bayesian model with a general multivariate normal density in the GP system is investigated. Specifically, the following research objectives will be investigated.

- Whether there are differences between using all basic features and randomly selecting a set of features in a Bayesian function.

- Whether using the simple Bayesian model as a terminal can achieve better performance than using the Bayesian model as a function.

- Whether the evolved Bayesian programs can be reasonably interpreted.

\subsection{Organisation}

In the remainder of the paper, Section 2 describes relevant background on edge detection and using GP for edge detection. Section 3 introduces a Bayesian GP system further developed from our previous work [14] to construct high-level features. Section 4 presents the design of the experiments. After giving the results in Section 5 with discussions, Section 6 provides further

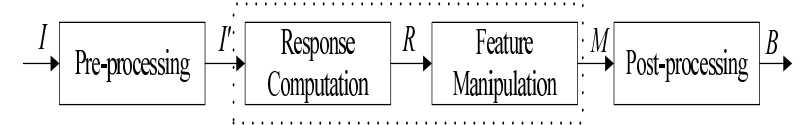

Fig. 1 General edge detection flow.

discussions. Finally, Section 7 draws conclusions and suggests future work directions.

\section{Background}

This section gives background on edge detection and discusses the existing work using GP for edge detection.

\subsection{Edge Detection}

Typically, edge detection includes three stages: pre-processing, feature extraction and post-processing [30]. A general process flow for edge detection is shown in Fig. 1. Given an image $I$, pre-processing techniques will use $I$ as input and generate an intermediate result $I^{\prime}$. In the pre-processing stage, noise and textures will usually be suppressed with little influence on edges. The second stage is to extract edge features, including computing responses on pixels (feature values) and manipulating features. In the process of response computation, edge responses, such as image derivatives [5], are employed. Based on different directional derivatives, a set of features $R$ is obtained. Note that it is possible to combine the pre-processing stage and the response computation stage together. For example, image Gaussian gradients include image gradients and Gaussian filtering [5]. In the process of manipulating edge features, feature selection [6] and further feature construction [17, 28] might be used to generate a set of features $M$. In edge detection, the process of extracting edge features is perhaps the most important. In the post-processing stage, marking edge points, thinning edges, linking broken edge points, and deleting stand-alone edge points are usually used. A final binary edge map $B$ is obtained after finishing post-processing operations. Generally, preprocessing and post-processing can be routinely applied to different edge feature extraction techniques.

To measure an edge feature based on ground truth, the feature is used to obtain final binary edge maps after applying thresholding techniques and post-processing techniques. For example, the $F$-measure technique [28] has been used to evaluate edge detectors and boundary detectors. Since common post-processing techniques, such as thinning operations [26] and non-maximum suppression [5], can generally follow most feature extraction approaches, it is also important to evaluate the features 
without non-maximum suppression and other specific post-processing techniques [29].

\subsection{Local Features}

A local moving window is usually used to extract edge features for each pixel. Local features are usually based on moving windows. The local rotation-invariant features used in this paper are introduced as follows.

\subsubsection{Image Gaussian Gradient}

Differentiation-based techniques are popular in edge feature extraction. For example, the image derivative [5, 17] and the local histogram derivative [28] have been used for extracting edge features. Horizontal derivatives of an image are used to detect vertical edges in the image. For example, to use a feature to detect vertical edges, the horizontal derivative of a Gaussian filter is defined in Equation (1), where $\sigma$ is the parameter scale of the Gaussian filter, and $(u, v)$ is the position of a neighbour relative to a centre discriminated pixel. Equation (2) describes the vertical derivative of a Gaussian filter. Given a direction $\theta=0^{\circ}$ or $90^{\circ}$, the relative image derivative $T_{g g, \theta}(x, y)$ is obtained after the convolution of image $I$ is performed with the derivative $g_{\theta}(u, v)$. Equation (3) describes the image Gaussian derivative $T_{g g, \theta}(x, y)$. Equation (4) defines the image Gaussian gradient $T_{g g}$. Here, $T_{g g}$ combines $T_{g g, 0^{\circ}}(x, y)$ and $T_{g g, 90^{\circ}}(x, y)$ as a rotation-invariant feature.

$$
\begin{aligned}
g_{0^{\circ}}(u, v) & =-\frac{u}{2 \pi \sigma^{4}} \exp \left(-\frac{u^{2}+v^{2}}{2 \sigma^{2}}\right) \\
g_{90^{\circ}}(u, v) & =-\frac{v}{2 \pi \sigma^{4}} \exp \left(-\frac{u^{2}+v^{2}}{2 \sigma^{2}}\right) \\
T_{g g, \theta}(x, y) & =g_{\theta}(u, v) \circledast I(x, y) \\
T_{g g}(x, y) & =\sqrt{T_{g g, 0^{\circ}}^{2}(x, y)+T_{g g, 90^{\circ}}^{2}(x, y)}
\end{aligned}
$$

\subsubsection{Image Histogram Gradient}

Objects normally have different histograms. Image local histogram derivatives have been applied to edge detection [28]. To detect pixel $(x, y)$ as an edge point at direction $\theta$, the image local histogram derivative around this pixel is defined in Equation (5). Here, a separate line with direction $\theta$ over pixel $(x, y)$ is used to divide the pixel's neighbours in a local area into the left and right groups. The values of these neighbours are binned. In bin $i, l_{\theta, i}$ is the number of occurrences of the neighbours in the left group, and $r_{\theta, i}$ is the number of occurrences of the neighbours in the right group. The local histogram gradient $T_{h g}$ is defined in Equation (6). Note that $h_{\theta}(x, y) \geq 0$ and $T_{h g}$ is the sum of $h_{\theta}(x, y)$ in a set of possible directions.

$$
\begin{aligned}
h_{\theta}(x, y) & =\frac{1}{2} \sum \frac{\left(l_{\theta, i}-r_{\theta, i}\right)^{2}}{l_{\theta, i}+r_{\theta, i}} \\
T_{h g} & =\sum_{\theta} h_{\theta}(x, y)
\end{aligned}
$$

\subsubsection{Normalised Standard Deviation}

In the literature, the normalised standard deviation is seldom employed as a feature for edge detection. Generally, it is used as an the indication of the image quality [32]. Equation (7) describes the local normalised standard deviation $T_{s d}(x, y)$. Here $\operatorname{Mean}(x, y)$ is the mean of the pixel intensities in a local area around pixel $(x, y)$, and $S D(x, y)$ are the standard deviation of the pixel intensities in the local area. The local area is typically a small moving window. Note that image rotation does not affect $T_{s d}(x, y)$ because the local area is rotation-invariant.

$T_{s d}(x, y)=\frac{S D(x, y)}{\operatorname{Mean}(x, y)}$

The notion of edges is the result of common human experience rather than a formal mathematical definition $[30,27]$. The local features from derivatives include high responses on non-edge points which are affected by noise or textures. For instance, Fig. 2 shows the three different edge features $T_{g g}, T_{h g}$ and $T_{s d}$ applied to an image from the Berkeley Segmentation Dataset (BSD) [28]. Fig. 2 (b) has problems in area 1 where it gives inappropriately strong responses on non-edge points and weak responses on edge points, area 2 where there is a lot of noise interference, and area 3 where edge points are not detected. Fig. 2 (c) shows better responses on the edges in area 1 than Fig. 2 (b), but has a problem in area 2 of noise interference, and only includes a few edge points in area 3 with many false alarms. Fig. 2 (d) also has a problem in area 1 where it gives too weak responses to the edge points, but is only weakly affected by noise in area 2 , and includes partial edges in area 3 . In order to obtain suitable edge features for desired edge outputs, a method to extract good edge features efficiently and effectively is required.

\subsection{Related Work on GP for Edge Feature Construction}

The existing limited work in edge detection using GP mainly focuses on construction of low-level edge features. Also, GP has been used to construct composite edge features. 


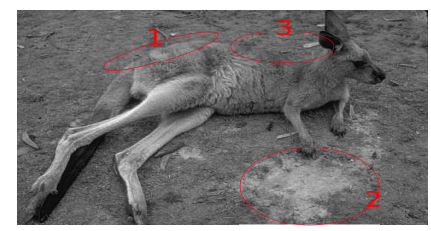

(a)69020.jpg

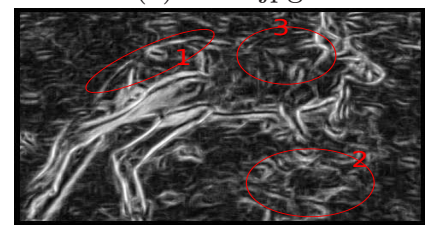

(c) $T_{h g}$

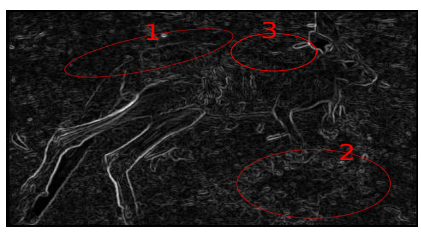

(b) $T_{g g}$

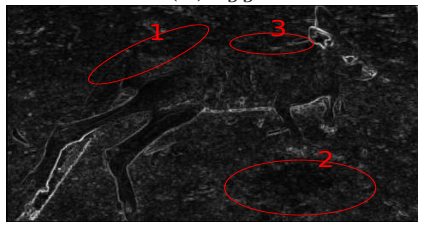

(d) $T_{s d}$
Fig. 2 Detected image based on Gaussian gradients $T_{g g}$, histogram gradients $T_{h g}$ and normalised standard deviations $T_{s d}$.

\subsubsection{Low-level Edge Feature Construction}

In the construction of low-level edge features, raw pixel values are combined together by a general formula to indicate whether pixels belong to edge points or nonedge points.

For example, GP has been used to approximate a target edge detector based on the responses of the target edge detector, such as the Sobel edge detector [20, 18] and the Canny edge detector [8]. Also, GP evolved specific programs to approximate desired outputs from a set of different one-dimensional step edge signals and noise [19]. These evolved programs were used to design one-dimensional filters to extract edge features. Like regression problems, GP is used for approximation of desired outputs, such as outputs of existing detectors or desired responses on signals. However, the desired outputs are obtained based on the understanding of edge responses. Only limited edge information can be used to construct edge features when GP is employed to approximate desired edge responses.

GP has also been used to extract edge features based on detection accuracy. Edge detection is considered as a special binary classification. Each pixel is discriminated as an edge point or a non-edge point. Similar to the four macros for searching a pixel's neighbours suggested by Poli [31], our previous work has employed search operators to select pixels to construct edge detectors $[10,12]$. Pixels in a $13 \times 13$ window were selected to extract edge features by GP using ground truth [38]. 64-bit digital circuits as programs were evolved by GP from artificial images [16]. In [36], morphological operators erosion and dilation were used as the terminal set and the detectors evolved by GP classify pixels as edge points or non-edge points. When GP is used to extract edge features based on ground truth, the prior domain knowledge on edges is weak. However, low-level edge features usually need to be further improved.

\subsubsection{Composite Edge Feature Construction}

To detect object boundaries, some image operators were utilised to combine high-level detectors by GP [22]. A rotation-variant feature evolved by GP in [22] was used to train a logistic regression classifier with texture gradients. However, only one composite feature (combined with texture gradients) in [22] was presented to compete with other edge and contour detectors. The variant feature is based on multiple directions, which leads to high computational cost. In addition, the GP feature needs to be combined with other existing approaches to perform boundary detection. In our previous work [11], three rotation-invariant basic features have been constructed into composite features. The composite features constructed by GP have the advantages from the basic features, and avoided some disadvantages from them. In [11], the composite features from GP are better than the combination of a Bayesian model. How to effectively combine basic features still needs to be investigated. In additional, GP was used to design programs which start at a pixel and then "walk" to find edge points [4].

\section{The Approach}

In order to employ existing techniques to combine basic features into composite features, functions or terminals based on existing techniques are designed in the proposed GP system. The function set includes functions implementing a Bayesian model and general algebraic operators. The terminal set includes not only basic features, but also a simple Bayesian model.

\subsection{Bayesian Function and Terminal}

Edge detection is considered here as a binary classification problem. Let $j=0$ be the class "non-edge point", $j=1$ be the class "edge point", and $x$ be a $d$-component vector-valued random variable. $P_{j}$ is the prior probability for the class non-edge point or edge point. Let $p_{x \mid j}$ be the state-conditional probability density function for $x$ (given $j=0$ or 1 being the true state). The posterior probability $p_{j \mid x}$ can be estimated from $p_{x \mid j}$ by Bayesian inversion (Equation (8)) [7]. Here the conditional probability density function $p_{x \mid j}$ is estimated by the general multivariate normal density (see Equation (9)), where, $\hat{\mu}_{j}$ is the $d$-component sample mean vector for class $j$, $\hat{\Sigma}_{j}$ is the $d$-by- $d$ sample covariance matrix, $\left|\hat{\Sigma}_{j}\right|$ is its 
determinant and $\hat{\Sigma}_{j}^{-1}$ is its inverse, and $\left(x-\hat{\mu}_{j}\right)^{T}$ is the transpose of $x-\hat{\mu}_{j}$. Note that $\left|\hat{\Sigma}_{j}\right|$ might be 0 . To avoid this situation, each diagonal element in $\hat{\Sigma}_{j}$ has a very small tolerance value $\epsilon=1.0 e-12$ added to it.

$p_{j \mid x}=\frac{p_{x \mid j} P_{j}}{\sum_{k=0}^{1} p_{x \mid k} P_{k}}$

$p_{x \mid j}=\frac{1}{(2 \pi)^{\frac{d}{2}}\left|\hat{\Sigma}_{j}\right|^{\frac{1}{2}}} \exp \left(-\frac{1}{2}\left(x-\hat{\mu}_{j}\right)^{T} \hat{\Sigma}_{j}^{-1}\left(x-\hat{\mu}_{j}\right)\right)$

In order to improve the performance of the standard Bayesian model, a Bayesian function is defined in Equation (10). Here, $s$ is the function argument, and $x$ is one of the possible combinations from the basic features $T_{g g}, T_{s d}$, and $T_{h g}$. The function argument $s$ is one basic feature or a subtree. Since the class edge point is the major class in edge detection, the output is a onedimensional variable based on the posterior probability of the class edge point. In edge detection, multiple outputs are not necessary, but the function can support multiple outputs, which is a potential future application to multiple classification problems.

$b_{1 \mid x}(s)=\frac{p_{1 \mid x, s}}{\sum_{j=0}^{1} p_{j \mid x, s}}$

The range of the output for the function $b_{1 \mid x}(s)$ is from 0 to 1 , and a composite feature from the simple Bayesian model [7] can be described as $b_{1 \mid x_{1}}\left(T_{h g}\right)$, where $x_{1}=\left\{T_{g g}, T_{s d}\right\}$. Note that the argument $s$ of the Bayesian function $b_{1 \mid x}$ can be the output of another $b_{1 \mid x}$, such as $b_{1 \mid x}\left(b_{1 \mid x}(s)\right)$.

Therefore, the Bayesian function has three behaviours: (1) selecting basic features; (2) combining basic features and $s$ (possibly as an intermediate combination); and (3) giving edge responses after estimating the relevant multivariate normal density.

In order to check whether the simple Bayesian model as a terminal is good to combine basic edge features, $b_{1 \mid x_{1}}\left(T_{h g}\right)$ is also considered as a terminal. Here $x_{\text {bayes }}$ indicates $b_{1 \mid x_{1}}\left(T_{h g}\right)$ used as a terminal. Since $x_{\text {bayes }}$ combines different basic edge features, the GP system allows a tree only consisting of a single terminal, e.g., $x_{\text {bayes }}$ (the simple Bayesian model) as a complete program.

\subsubsection{General Algebraic Operators}

In order to enrich the evolved Bayesian programs, ordinary algebraic operators need to be added into the function set for constructing composite features. However, the constructed composite features are expected to represent soft edge maps. A sparse and large range of the values of a constructed feature is not suitable to represent soft edge maps. Our previous work [13] has addressed this problem. Also, it is found that directly using observations of evolved programs with normal algebraic operators was not good to construct soft edge maps [11]. The transformation by a function used in the Bayesian GP system requires the mapped values to be located in a suitable range. In general, a feature, which is used to represent soft edge maps, is normalised from 0 to 1 . In the simple Bayesian model, the output of a Bayesian function or $x_{\text {bayes }}$ is from 0 to 1 . All operations in the Bayesian GP system are required to map their inputs into the space from 0 to 1 .

For linear operations, two general algebraic operators $\{\oplus, \ominus\}$ are added into the function set. The operation of $\oplus$ is defined in Equation (11), and the operation of $\ominus$ is defined in Equation (12). Here, $s_{a}$ and $s_{b}$ are the arguments of the operators $\oplus$ and $\ominus$, and the operator $\ominus$ is the absolute value of difference of $s_{a}$ and $s_{b}$. The ranges of $s_{a}$ and $s_{b}$ are from 0 to 1 , so the range of the outputs of both operators is also from 0 to 1 .

$s_{a} \oplus s_{b}=\frac{s_{a}+s_{b}}{2}$

$s_{a} \ominus s_{b}=\left|s_{a}-s_{b}\right|$

Non-linear general algebraic operators could be used in the function set. Since this paper only focuses on the Bayesian model, the application of using different general algebraic operators will be investigated in the future work.

\subsection{The Other Terminals}

The basic features are considered as terminals and they are normalised from 0 to 1 . Note that random constants are not included in the terminal set because a Bayesian program estimates the relevant multivariate density to construct a composite feature, rather than directly using its observations as a feature. Also, the outputs for all operations in the GP system are located in the range from 0 to 1 . The operator $\oplus$ will automatically adjust the scales for its input (such as Equation (11)) so that its output is still located in the range from 0 to 1.

\subsection{Fitness Function}

From the set of terminals and functions, the range of outputs of a program is from 0 to 1 , which is directly considered as the value of a composite feature. The aim of the new constructed features is to detect as many true edge points as possible, so the fitness function Fit is defined in Equation (13). Here, recall $p_{r e c}$ is the number of pixels on the edges correctly detected as a proportion of the total number of pixels on the edges, and 
specificity $p_{\text {spe }}$ is the number of pixels not on the edges correctly detected as a proportion of the total number of pixels not on the edges. Recall $p_{r e c}$ and specificity $p_{\text {spe }}$ are calculated when a threshold is used to discriminate outputs of a program as edge points or non-edge points.

$F i t=\frac{2 p_{\text {rec }} p_{\text {spe }}}{p_{\text {rec }}+p_{\text {spe }}}$

Since there are no random constants in the terminal set and the range of observations of evolved programs is restricted from 0 to 1 , multiple thresholds are used to find the maximum of Fit. Here, only three thresholds are employed to find the maximum value of Fit as the fitness for the relevant program, aiming at evolving programs with high contrast edge responses. The three thresholds are $0.25,0.5$ and 0.75 . In order to check whether three thresholds are enough for evaluating evolved programs, 30 thresholds $\left(\frac{i}{31}, i=\right.$ $1,2, \ldots, 30)$ are also used to find maximum Fit. To distinguish the two different settings on Fit, Fit 3 indicates Fit with the three thresholds, and Fit 30 indicates Fit with the 30 thresholds.

Different from our previous work [14], the Bayesian function is considered not only a function node, but also a terminal node in the Bayesian GP system. Also, $F i t$ is allowed to use different numbers of thresholds. To further investigate the Bayesian function, there are different settings for the function set and the terminal set in the GP system. The details of these settings are given in the next section.

\section{Experiment Design}

We now describe the benchmark image dataset used in this paper, also give the settings for the experiments.

\subsection{Image Datasets}

A natural image dataset, namely the Berkeley Segmentation Dataset (BSD) [28], is employed for edge detection. Each image in the BSD dataset has $481 \times 321$ pixels and its own ground truth from different human observations. The ground truth are combined from five to ten persons as graylevel images for fairness of judgement of edges. This dataset has been popularly used for boundary detection [24] and image segmentation [1]. The training dataset and the test dataset are separated. There are 200 images in the training dataset and 100 different images in the test dataset. Fig. 3 shows six images from the training dataset and their ground truth. Note that the widths of some true edges are more than
Table 1 Settings for terminals and functions in the Bayesian GP system.

\begin{tabular}{l|c|c|c}
\hline \multicolumn{2}{c|}{ Setting } & Terminals & Functions \\
\hline Bayesian & Set $_{b f, \text { rand }}$ & $\{x\}$ & $\left\{b_{1 \mid x}, \oplus, \ominus\right\}$ \\
Function & Set $_{b f, \text { all }}$ & $\{x\}$ & $\left\{b_{\left.1 \mid x_{\text {all }}, \oplus, \ominus\right\}}\right.$ \\
\hline \multirow{2}{*}{ Bayesian } & Set $_{b t}$ & $\left\{x, x_{\text {bayes }}\right\}$ & $\{\oplus, \ominus\}$ \\
Terminal & Set $_{b t, \text { all }}$ & $\left\{x, x_{\text {bayes }}\right\}$ & $\left\{b_{\left.1 \mid x_{\text {all }}, \oplus, \ominus\right\}}, \oplus, \ominus\right\}$ \\
& Set $_{\text {bt }, \text { rand }}$ & $\left\{x, x_{\text {bayes }}\right\}$ & $\left\{b_{1 \mid x}, \oplus, \ominus\right\}$ \\
\hline Full Set & Set $_{\text {full }}$ & $\left\{x, x_{\text {bayes }}\right\}$ & $\left\{b_{1 \mid x}, b_{1 \mid x_{\text {all }}}, \oplus, \ominus\right\}$ \\
\hline
\end{tabular}

one pixel because of the combinations of several human observations. The pixels with graylevel 0 (dark) in the ground truth are non-edge points, and the others are edge points. Pixels from the training images are sampled as training dataset, which is the same as in [11]. The settings of the three basic features are the same as in [11] as well.

The true edges in the BSD dataset come from human segmentation results, they are more closer for boundary detection, and not exact for edge detection. However, boundaries are usually extracted based on the edges detected by an edge detector [30, 27]. The edges detected by an edge detection algorithm are affected the performance of detected boundaries. One way in [34] has been proposed to evaluate edge detection through boundary detection. Also, following in the suggestion of directly evaluating edge features in [29], we directly use the ground truth as the desired outputs to do the performance evaluation on extracted edge features.

\subsection{Experiment Settings}

Here, $x_{a l l}$ indicates that all basic features $\left(T_{g g}, T_{s d}\right.$ and $\left.T_{h g}\right)$ are used in the terminal set. Also, $b_{1 \mid x_{a l l}}$ indicates that the Bayesian function always includes all basic features, and $b_{1 \mid x}$ indicates that the Bayesian function randomly selects one of all combinations of the basic features. In order to investigate the influence of different terminals and functions, different settings for the terminal and function sets are listed in Table 1.

Firstly, the Bayesian functions are investigated. Setting Set $_{b f, \text { rand }}$ automatically selects basic features to construct composite features. Setting Set $_{b f, a l l}$ restricts that all basic features must be included in a Bayesian function. The purpose for different settings on Bayesian functions is to find the difference between a large space (using $b_{1 \mid x}$ to include possible combinations of basic features) and a narrow space (must use all basic features) for feature construction. Secondly, three settings $S_{e t} t_{b t}$, Set $t_{b t, a l l}$ and $S e t_{b t, \text { rand }}$ are used to investigate the relationship between the terminal $x_{\text {bayes }}$ and the functions 


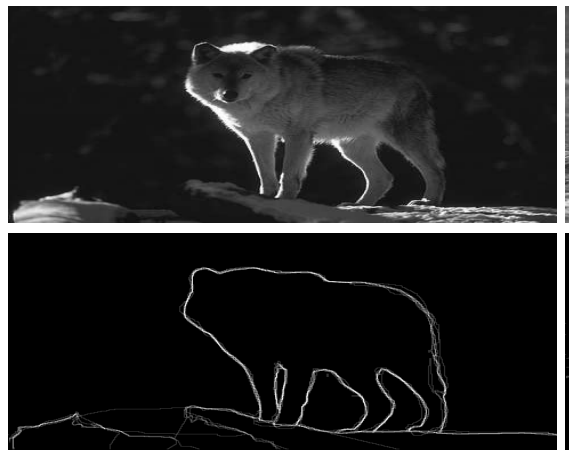

(a) 42078
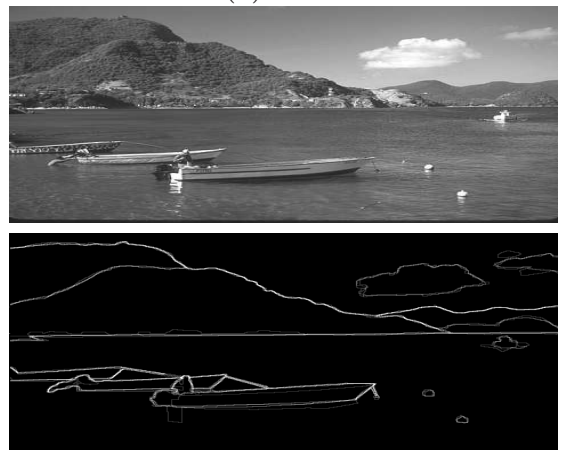

(d) 68077

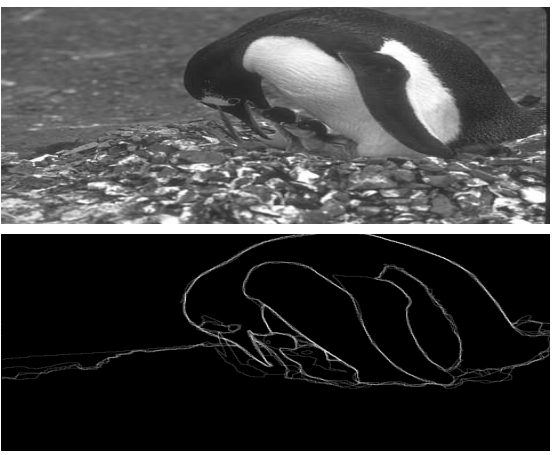

(b) 106020

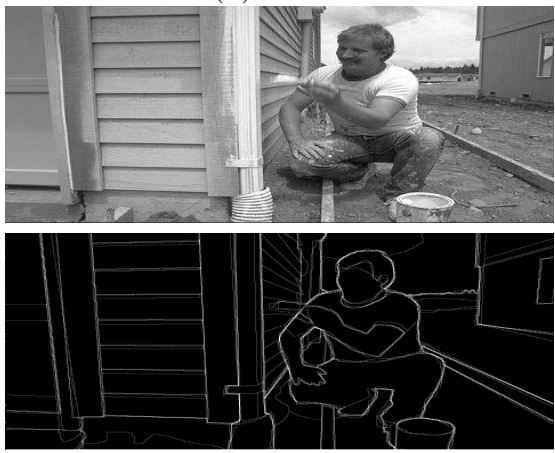

(e) 23080

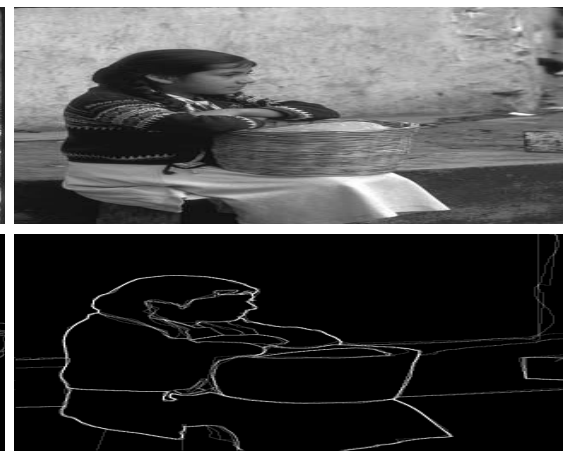

(c) 23025

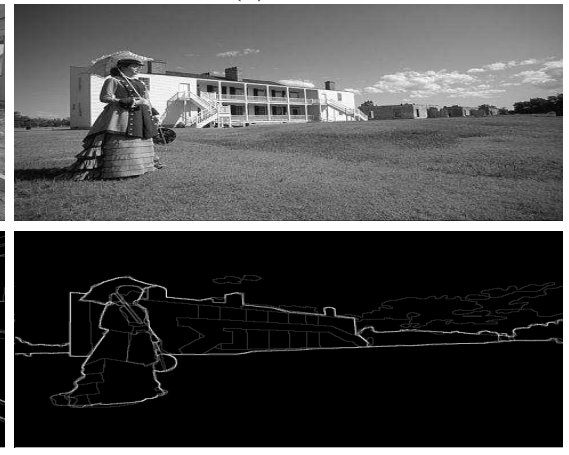

(f) 216053

Fig. 3 Six example training images from the BSD dataset and their ground truth.

$b_{1 \mid x}$ and $b_{1 \mid x_{a l l}}$. Lastly, a full set of terminals and functions is given in setting Set $_{\text {full }}$.

The parameter values for GP are: population size 50; maximum generations 50; maximum depth (of a program) 5, probabilities for mutation 0.15 , crossover 0.80 and elitism (reproduction) 0.05 . These values are chosen based on common settings and initial experiments [10]. Note that a Bayesian program has some ability to combine the basic features after estimating sample means and standard distributions in the program. A larger population and a larger number of generations are not necessarily required.

Each experiment is repeated 30 independent runs. Here, the test performance employs the F-measure technique [28]. The F-measure technique combines recall $p_{r e c}$ and precision $p_{\text {pre }}$, and it is defined in Equation (14). Here, precision $p_{\text {pre }}$ is the number of pixels on the edges correctly detected as a proportion of the total number of pixels detected as edge points, and $\alpha$ is a factor from 0 to $1 . \alpha$ is usually set to $0.5[28,6]$, which is also used in this paper. Based on thresholds $\frac{k}{52}, k=1,2, \ldots, 51$, the maximum $F_{\max }$ of $F$ values is considered as the test performance of a constructed feature.

$$
F=\frac{p_{\text {rec }} p_{\text {pre }}}{\alpha p_{\text {rec }}+(1-\alpha) p_{\text {pre }}}
$$

\section{Results}

This section provides experiment results with discussions. The results from $\mathrm{Fit}_{3}$ will be given, then the results are compared with existing techniques. A comparison between $\mathrm{Fit}_{3}$ (three thresholds) and $\mathrm{Fit}_{30}(30$ thresholds) will be conducted. After showing detected images from $\mathrm{Fit}_{3}$ and $\mathrm{Fit} t_{30}$, an example evolved Bayesian program will be interpreted.

\subsection{Overall Results From $\mathrm{Fit}_{3}$}

Table 2 gives the means, standard deviations (s.d.), the maximum ("Max"), and the minimum ("Min") of $F_{\max }$ values of the evolved programs for the six settings when fitness function $\mathrm{Fit}_{3}$ is used. The "t-test" column reports the $p$-values obtained from the comparisons between the relevant results (first column as the first group) and the results from Set $_{b f, \text { rand }}$ (as the second group) by using two-sample $t$-tests; and "MWW" reports the $p$-values obtained from the comparisons between the relevant results and the results from $S_{\text {et }} t_{b f, \text { rand }}$ by using Mann-Whitney-Wilcoxon tests [25].

From an overall view, setting $S_{\text {et }}$, rand has the highest mean and the maximum $F_{\max }$. It seems that setting $S_{e t} t_{b, \text { rand }}$ has the best test performance on the BSD test images. In regards of the $p$-values from the 
Table 2 Test performance $F_{\max }$ for the six settings using fitness function $\mathrm{Fit}_{3}$ on the BSD test image dataset.

\begin{tabular}{c|ccccc}
\hline Setting & Mean \pm s.d. & Max & Min & $t$-test & MWW \\
\hline Set $_{b f, \text { rand }}$ & $0.5591 \pm 0.0150$ & 0.5847 & 0.5199 & & \\
Set $_{b \text {, all }}$ & $0.5423 \pm 0.0053$ & 0.5504 & 0.5349 & $0.0000 \downarrow$ & $0.0000 \downarrow$ \\
Set $_{b t}$ & $0.5391 \pm 0.0000$ & 0.5391 & 0.5391 & $0.0000 \downarrow$ & $0.0000 \downarrow$ \\
Set $_{b t, \text { all }}$ & $0.5381 \pm 0.0137$ & 0.5664 & 0.5102 & $0.0000 \downarrow$ & $0.0000 \downarrow$ \\
Set $_{b t, \text { rand }}$ & $0.5513 \pm 0.0107$ & 0.5733 & 0.5277 & $0.0278 \downarrow$ & 0.0543 \\
Set $_{\text {full }}$ & $0.5513 \pm 0.0181$ & 0.5841 & 0.5102 & 0.0798 & 0.0603 \\
\hline
\end{tabular}

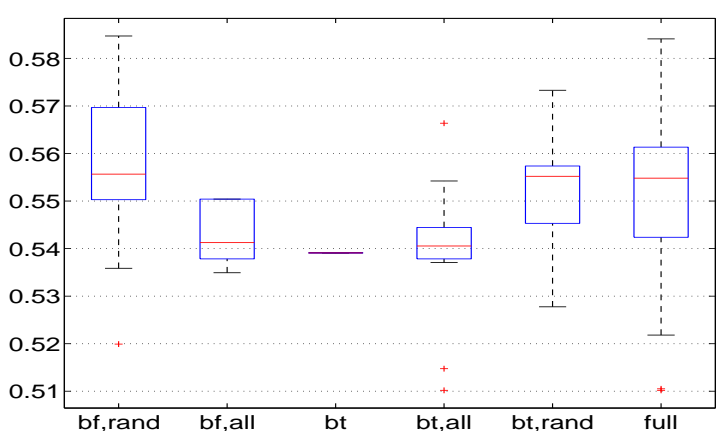

Fig. 4 Boxplots for the $F_{\max }$ values of the results (30 replications) from the six settings using fitness function $\mathrm{Fit}_{3}$ on the 100 BSD test images.

$t$-tests, the results from $S_{\text {et }}$, rand are significantly better than the results from the other settings, except for Set $_{f u l l}$. From the Mann-Whitney-Wilcoxon tests, the results from $S e t_{b f, \text { rand }}$ are also significantly better than the results from $S_{e t} t_{b f, a l l}, S_{b t}$ and $S_{b e} t_{b t, a l l}$. Since only settings $S_{\text {et }}$,rand, Set $t_{f u l l}$ and Set $_{b t, \text { rand }}$ include $b_{1 \mid x}$, it seems that $b_{1 \mid x}$ is important to construct high-level features for performance improvement when fitness function Fit 3 is used.

Fig. 4 reveals the $F_{\max }$ values of the results from the six settings using $\mathrm{Fit}_{3}$. Here the box labels are the indices of the six settings, and each box represents the relevant setting. From these boxplots, the results from Set $_{b t}$ are the same. From Fig. 4 and Table 2, a few evolved programs from settings Set $_{b, \text {, rand }}$, Set $t_{b t, a l l}$ and Set $_{\text {full }}$ have very bad test performance, and their $F_{\max }$ values are less than 0.52 . From an overall view, all settings, expect for $S_{e t}$, have at least half of their evolved programs with $F_{\max }$ values larger than 0.54 . From our previous work in [11], the highest $F_{\max }$ among the three basic features is 0.5434 , and a simple Bayesian method has $F_{\max }=0.5302$, which is obviously lower than most of the evolved programs from the six settings here, except for $S_{e t}$. Therefore, GP can effectively evolve Bayesian programs to improve detection performance when fitness function Fit only uses the three thresholds to find the maximum value.

\subsubsection{Bayesian Function}

From the comparison between $\operatorname{Set}_{b f, \text { rand }}$ (including $b_{1 \mid x}$ ) and $S_{e} t_{b f, a l l}$ (including $b_{1 \mid x_{a l l}}$ ), the latter is significantly worse. If using $\mathrm{Fit}_{3}$, the Bayesian program including all three basic features is not good to find good Bayesian programs. From the boxplots in Fig. 4, the test performances of the evolved programs from Set $_{b f, a l l}$ are located in a narrow range. However, the test performance of the evolved programs from Set $_{b f \text {, rand }}$ spreads over a larger range. A reason is that function $b_{1 \mid x_{\text {all }}}$ always includes the three basic features. After estimating an evolved program including $b_{1 \mid x_{a l l}}$, the test performance is strongly affected by the combination of three basic features (as a Bayesian model). Note that fitness function $\mathrm{Fit}_{3}$ only uses three thresholds to find the maximum value, and a Bayesian model with three basic features has high contrast responses for edge points. However, function $b_{1 \mid x}$ randomly selects basic features, it is possible that only one basic feature is selected and the evolved program has high test performance. Since the combination of three basic features using $b_{1 \mid x}$ are varied and the evolved programs from using $b_{1 \mid x_{a l l}}$ always include all basic features, an evolved program from using function $b_{1 \mid x}$ may have lower $F_{\max }$ than the evolved programs from using function $b_{1 \mid x_{a l l}}$. From the minimum value of test performance $F_{\max }$ in Table 2 and Fig. 4, a bad evolved program exists in setting Set $_{b f, \text { rand }}$, but there are no outliers in setting Set $_{b f, a l l}$.

Therefore, when Fit only uses the three thresholds to find the maximum value as the fitness of an evolved program, the evolved program with $b_{1 \mid x_{\text {all }}}$ is strongly connected to the combination of all basic features and the test performance is located in a stable range; but evolved programs with $b_{1 \mid x}$ have flexible structures to combine basic features, which brings good test performance on some evolved programs in most cases, at the same time, leads to bad test performance on a few evolved programs.

\subsubsection{Bayesian Terminal}

When $x_{\text {bayes }}$ is added into the terminal set, all evolved programs from Set $b t$ using $\mathrm{Fit}_{3}$ are equal to the single 
Table 3 Comparison between the results from Set $_{b, \text { rand }}$ using $\mathrm{Fit}_{3}$, image Gaussian gradients $T_{g g}$, normalised standard deviations $T_{s d}$, histogram gradients $T_{h g}$, Sobel edge detector and the simple Bayesian terminal $x_{\text {bayes }}$ on the 100 BSD test images.

\begin{tabular}{c|cc}
\hline & $F_{\max }$ & $p$-value \\
\hline Set $_{b f, \text { rand }}$ & $0.5591 \pm 0.0150$ & \\
$T_{g g}$ & 0.5153 & $0.000 \downarrow$ \\
$T_{s d}$ & 0.4968 & $0.000 \downarrow$ \\
$T_{h g}$ & 0.5434 & $0.000 \downarrow$ \\
Sobel & 0.4832 & $0.000 \downarrow$ \\
$x_{\text {bayes }}$ & 0.5391 & $0.000 \downarrow$ \\
\hline
\end{tabular}

node tree $x_{\text {bayes }}$. It seems that $\mathrm{Fit}_{3}$ could not find any better combination from $x_{\text {bayes }}$ and the three basic features without using Bayesian functions, compared with $x_{\text {bayes. }}$. Note that the test performance $F_{\max }$ on $x_{\text {bayes }}$ is a bit higher than the simple Bayesian model in [11]. The change is caused by $x_{\text {bayes }}$ adding tolerance $\epsilon$ in $\hat{\Sigma}_{j}$.

After adding Bayesian functions, the results from $S_{\text {Set }}$, all are similar to the results from Set $_{b f, a l l}$, and the results from $S_{\text {et }}$,rand are similar to the results from Set $_{b f, \text { rand }}$. It is surprising that there are two evolved programs from Set $t_{b f, \text { all }}$ with $F_{\max }$ values less than 0.52. In Set $_{b f, a l l}, b_{1 \mid x_{a l l}}$ and $x_{\text {bayes }}$ include the three basic features, but there are still two programs with bad test performance. From the boxplots in Fig. 4, the terminal $x_{\text {bayes }}$ might be not good for constructing high-level features when fitness function $\mathrm{Fit}_{3}$ is used. This suggests that directly combining the simple Bayesian model with all basic features might be not good for performance improvement.

\subsubsection{Combination}

When all functions and terminals are added into the GP system, namely using $S e t_{f u l l}$, the results from Set $_{\text {full }}$ are not significantly different from the results from setting Set bf,rand . It seems that adding $x_{\text {bayes }}$ and $b_{1 \mid x_{\text {all }}}$ in setting Set $_{b f, \text { rand }}$ does not affect the evolved programs when $\mathrm{Fit}_{3}$ is used. Although the search space for candidate solutions from Set $_{\text {full }}$ is larger than the space from $S_{e t} t_{b f, r a n d}$, GP can still effectively discover good programs to construct high-level features.

\subsection{Set $_{b f, \text { rand }}$ using $\mathrm{Fit}_{3}$ vs Existing Techniques}

Table 3 presents the comparisons between the results from Set $_{b f, \text { rand }}$ using $\mathrm{Fit}_{3}$ and the results from $T_{g g}, T_{s d}$, $T_{h g}$, the Sobel edge detector, and $x_{\text {bayes }}$. The $p$-values are obtained from the comparison between the relevant results (first row as the first group) and the results from

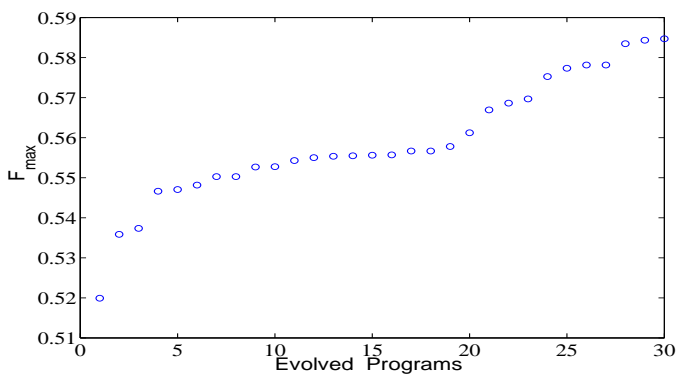

Fig. $5 F_{\max }$ values for the features constructed by the evolved 30 programs from GP using Set $_{b f, \text { rand }}$ and Fit 3 .

Set $_{b f, \text { rand }}$ using $\mathrm{Fit}_{3}$ (as the second group) when onesample $t$-tests are used. In additional, the $95 \%$ confidence interval (based on the $t$-test) for the evolved programs from $S_{e t} t_{b f, \text { rand }}$ is $(0.5533,0.5648)$. These results show that the features constructed by GP significantly improve the detection performance. However, the $F_{\max }$ value from $x_{\text {bayes }}$ is lower than the basic feature $T_{h g}$, so it seems that the simple combination does not improve the detection performance $\left(F_{\max }\right)$. Therefore, GP is effective for automatic construction of new features, and improves the performance of using the Bayesian model to combine basic edge features for edge detection.

In order to check the details of the performance on all evolved programs, Fig. 5 gives the relevant $F_{\max }$ values for all 30 evolved Bayesian programs from Set $_{b f, \text { rand }}$ with $\mathrm{Fit}_{3}$. Most of the evolved Bayesian programs have higher $F_{\max }$ than the best basic feature $T_{h g}$, and most of the evolved programs obtain good performance. However, the constructed features from three evolved programs are worse than $T_{h g}$ (with $F_{\max }$ ). The lowest $F_{\max }$ value of the constructed feature from the worst evolved program is only 0.52 . How to improve the performance on the worst evolved program will be a future work.

\subsubsection{Recall and Precision of an Evolved Program}

Fig. 6 shows different values of recall and precision for $T_{g g}, T_{s d}, T_{h g}, x_{\text {bayes }}$, and a program evolved by GP (with $F_{\max }=0.5847$ ). Here, "@" is the position for the $F_{\max }$. From the different thresholds, it is clear that the evolved program has higher precision than the others when recall is not too low. For $T_{g g}$, recall is high but precision is too low, and precision is high but recall is too low.

One interesting observation from Fig. 6 is that $x_{\text {bayes }}$ has a narrow range for recall and precision. A reason for this phenomenon is that the Bayesian Equation (8) gives very low probabilities for most non-edge points and very high probabilities for most edge points. Therefore, the change of the threshold does not strongly af- 
Table 4 Test performance $F_{\max }$ for the six settings using $F i t_{30}$ on the BSD test image dataset.

\begin{tabular}{c|cccccc}
\hline Setting & Mean \pm s.d. & Max & Min & t-test & MWW & Fit $_{3}$ \\
\hline Set $_{b f, \text { rand }}$ & $0.5754 \pm 0.0134$ & 0.5968 & 0.5509 & & & $0.0000 \uparrow$ \\
Set $_{b f, \text { all }}$ & $0.5707 \pm 0.0104$ & 0.5927 & 0.5531 & 0.1397 & 0.0726 & $0.0000 \uparrow$ \\
Set & $0.5706 \pm 0.0198$ & 0.5966 & 0.5087 & 0.2824 & 0.2697 & $0.0000 \uparrow$ \\
Set $_{b t, \text { all }}$ & $0.5706 \pm 0.0166$ & 0.5950 & 0.5250 & 0.2278 & 0.4705 & $0.0000 \uparrow$ \\
Set $_{b t, \text { rand }}$ & $0.5656 \pm 0.0230$ & 0.5946 & 0.4998 & 0.0518 & 0.1290 & $0.0053 \uparrow$ \\
Set $_{\text {full }}$ & $0.5684 \pm 0.0159$ & 0.5939 & 0.5087 & 0.0743 & 0.2697 & $0.0006 \uparrow$ \\
\hline
\end{tabular}

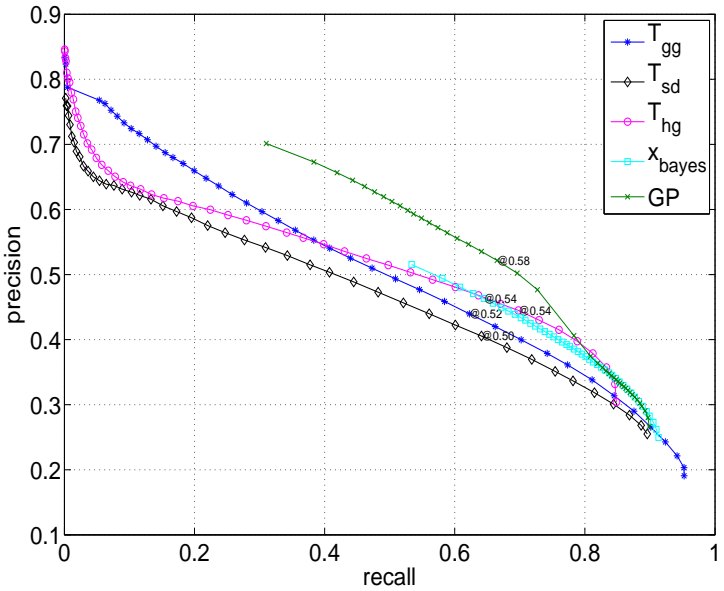

Fig. 6 Recall and precision for $T_{g g}, T_{s d}, T_{h g}, x_{\text {bayes }}$, and an evolved program.

fect the detection performance. Compared with the basic features, $x_{\text {bayes }}$ and the evolved program have very high recall values for many thresholds. It seems that the composite features from the Bayesian model can easily discriminate pixels as edge points or non-edge points with a set of thresholds. This is similar to the characteristics of edge responses (high contrast) on the composite features constructed by using Gaussian distribution estimation. Whether the multivariate density using other distributions can be used to obtain rich edge responses will be investigated in the future.

\subsection{Fitness Functions Fit $_{3}$ vs Fit $_{30}$}

Table 4 gives the means, standard deviations, maximum and minimum of $F_{\max }$ values for the six settings using $F_{i t}{ }_{30}$. Here, " $t$-test" indicates $p$-values obtained from the comparisons between the relevant results (setting in the first column as the first group) and the results from $S e t_{b f, \text { rand }}$ using two-sample $t$-tests, MWW indicates $p$ values from their comparisons with the Mann-WhitneyWilcoxon tests, and " $F i t_{3}$ " indicates $p$-values obtained from the comparisons between the results from Fit ${ }_{30}$ and $\mathrm{Fit}_{3}$ (using the same setting) with paired-sample $t$-tests. The paired-sample $t$-tests are used for the com- parisons between Fit $_{30}$ and Fit $_{3}$ because of the same initial population.

There are some interesting observations from Table 4 . Firstly, the test results from $F_{i t} t_{30}$ are significantly better than the results from $\mathrm{Fit}_{3}$. It seems that the evaluation based on three thresholds is not good to find good features (in terms of $F_{\max }$ ), although Bayesian functions or terminals give high contrast responses. A potential reason is that Bayesian outputs are a combination of basic features with the two general algebraic operators. Since combining the three basic features needs multiple thresholds to find the maximum Fit values, a program, including the combination of Bayesian subtrees and subtrees constructed by basic features and the two general algebraic operators, might give rich responses on edge points and non-edge points. After using 30 thresholds to find the maximum of Fit as fitness for evolved programs, subtrees constructed by basic features and the two general algebraic operators might strongly affect the fitness of the relevant program.

Secondly, when Fit $_{30}$ is used, there are no significant differences between the results from Set ${ }_{b f, \text { rand }}$ and the other settings. Using multiple thresholds is easier to find a threshold which is closer to the optimal threshold for Fit. Some evolved programs evaluated by $\mathrm{Fit}_{3}$ are not good, but they have good binary outputs by another threshold, not including in the thresholds used in $\mathrm{Fit}_{3}$. Since Fit 30 is more reasonable than $\mathrm{Fit}_{3}$ to evaluate programs' fitness, all settings have good results. From the $t$-tests and the MannWhitney-Wilcoxon tests, there is no influence from using the Bayesian node in the function set or the terminal set. The way of selecting the full set of basic features or randomly selecting basic features does not obviously affect the test performance of the evolved problems.

Thirdly, the maximum $F_{\max }$ in each setting is increased. It seems that the ability to find good programs to construct high-level features is improved after using $\mathrm{Fit}_{30}$.

Lastly, the worst evolved programs from settings Set $t_{b f, \text { rand }}$ and $S_{\text {et }}$ bf,all are improved obviously when $F_{i t} t_{30}$ replaces $F_{i t}$, but the worst evolved programs from settings $S_{e t}$, $S e t_{b t, \text { rand }}$ and $S_{\text {et }}$ full become even 
worse, in terms of $F_{\max }$. It is interesting that multiple thresholds do not improve the test performance of the worst evolved programs for the three settings. From each setting including $x_{\text {bayes }}$, the worst test performance from their results is lower than the test performance on each of the basic features. Therefore, the simple Bayesian model with all basic features might be not good to use as a terminal, and it is suggested that the Bayesian combination technique should only be used as a function to combine basic features.

\subsection{Detected Images}

Fig. 7 shows two example detected images from the three basic features, and the best evolved programs from $\mathrm{Fit}_{3}$ and $\mathrm{Fit}_{30}$ with setting Set $_{b f, \text { rand }}$. From the detected images, the detected images by $x_{\text {bayes }}$ and the evolved programs have obviously strong responses on true edge points, which is similar to $T_{h g}$. The strong responses on non-edge points from $T_{h g}$ are mostly suppressed by $x_{\text {bayes }}$ and the evolved programs. However, some non-edge points with high responses from $T_{h g}$ still have high responses in $x_{\text {bayes }}$. Comparing the responses from $T_{s d}$ and $T_{g g}$, most of the non-edge points with high responses from $T_{h g}$ in these two images are obviously decreased in the GP evolved programs. Therefore, the Bayesian GP system effectively constructs new composite features.

From the visual results detected by the program from $\mathrm{Fit}_{3}$, the strong responses on non-edge points from discontinuous background areas (such as $T_{h g}$ ) are decreased. Comparing with visual results from Fit $_{3}$, the responses on these non-edge points are further decreased in the two images detected by the evolved program from Fit $_{30}$. However, the weak responses on non-edge points from the background in the two detected images from Fit $_{30}$ are increased a bit. Since these increased responses are still obviously weaker than the responses on edge points, the influence can be neglected. Also, from the subjective view, the detected images from $\mathrm{Fit}_{30}$ is thinner than the detected images from Fit 3 . These observations suggest that the multiple thresholds in Fit can improve the ability of finding better programs to construct features.

Fig. 8 shows the image 69020 detected by the best

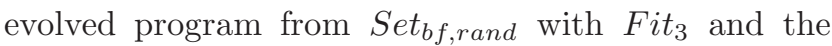
combination $x_{\text {bayes }}$. As can be seen, the detected image by the best evolved program from GP is improved in areas 1 and 2. Also, the responses on edge points are obviously higher than the non-edge points. Most of the true non-edge points have very weak responses (very dark) for this image, which is the same as the detection result from $x_{\text {bayes }}$. Although $x_{\text {bayes }}$ decreases responses on most of the non-edge points, it wrongly strengthens responses on a few non-edge points.

\subsection{Example Evolved Bayesian Program}

The best evolved Bayesian GP program from Set $_{b f, \text { rand }}$ with $\mathrm{Fit}_{3}$ is described by Equation (15), where $B G_{\text {bayes }_{1}}$ indicates an evolved Bayesian program, and subscript indices 0,1 and 2 represent the basic features $T_{g g}, T_{s d}$ and $T_{h g}$ (in $b_{x}$, abbreviated from $b_{1 \mid x}$ ), respectively. Function $b_{0,1}\left(T_{g g}\right)$ is used to construct a model with the multivariate including $T_{g g}$ and $T_{s d}$. Note that the proposed function accepts redundant variables, such as $x=$ $\left\{T_{g g}, T_{g g}\right\}$. From the first part $b_{0,1,2}\left(b_{0,1}\left(b_{0,1}\left(T_{g g}\right)\right)\right)$, the formula aims at finding true edge points detected by $T_{g g}$ and then constructing a feature with three basic variables. Since $T_{g g}$ has high recall, this first part should be good at finding true edge points. The second part $b_{2}\left(b_{2}\left(b_{2}\left(T_{h g}\right)\right)\right)$ only includes the basic feature $T_{h g}$. After repeating estimation on true edge points (using the second part), the responses for those non-edge points with high responses in $T_{h g}$ should be decreased. Since the output of the Bayesian function is based on the class edge point, the accuracy (precision) for the constructed feature should not be too low. Generally, the responses on clear boundaries (easily detected) are accurately given. Therefore, the second part possibly focuses on the boundary detection.

$$
B G_{\text {bayes }_{1}}=b_{0,1,2}\left(b_{0,1}\left(b_{0,1}\left(T_{g g}\right)\right)\right) \oplus b_{2}\left(b_{2}\left(b_{2}\left(T_{h g}\right)\right)\right)
$$

In order to visually present the characteristics from the two parts, Fig. 9 shows two example images detected by the two parts of the best evolved method. "First" means the images detected by the first part $b_{0,1,2}\left(b_{0,1}\left(b_{0,1}\left(T_{g g}\right)\right)\right)$, and "Second" for the second part $b_{2}\left(b_{2}\left(b_{2}\left(T_{h g}\right)\right)\right)$. From the two example detected images, the second part appears to be better than the first part to detect true edge points. Since the first part focuses on finding edge points (possibly with low precision), the combination of both parts gives high responses on boundaries and low responses on non-edge points. The test performances $\left(F_{\max }\right)$ on the first part and second part for the 100 BSD test images are 0.5231 and 0.5436 , and the complete $B G_{\text {bayes }_{1}}$ has $F_{\max }=$ 0.5847. Compared with $T_{h g}$, the second part does not improve the $F_{\max }$ value, but it decreases response magnitudes for non-edge points. Iteratively estimating a single feature might be helpful to improve detection performance, which is a future work.

In order to further check the performance of both parts, Fig. 10 reveals the details of recall and precision for the two parts of the evolved programs. From the 


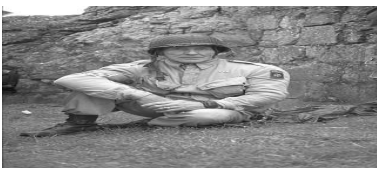

Image 376043

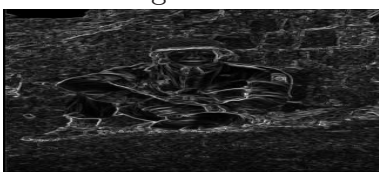

$T_{s d}$

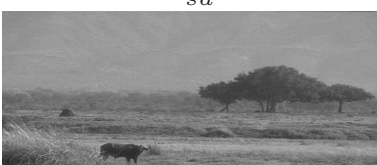

Image 296007

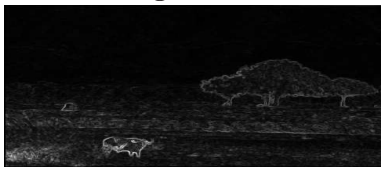

$T_{s d}$

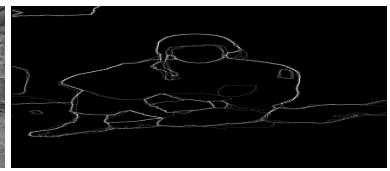

Ground Truth

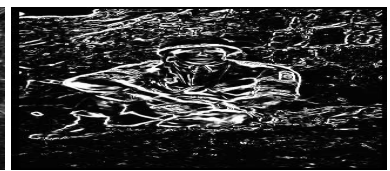

$x_{\text {bayes }}$

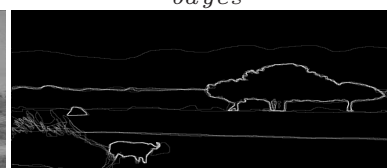

Ground Truth

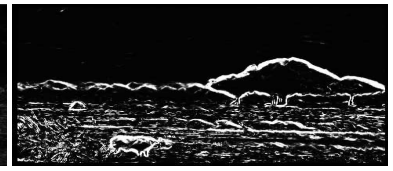

$x_{\text {bayes }}$

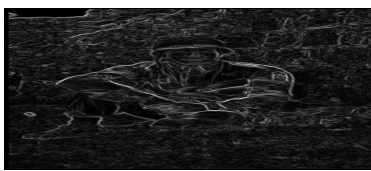

$T_{g g}$

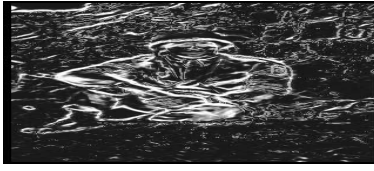

$\mathrm{Fit}_{3}$

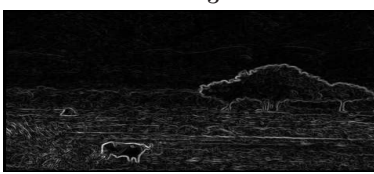

$T_{g g}$

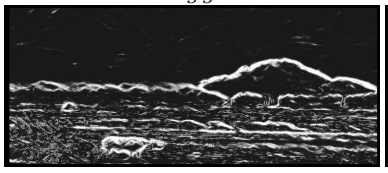

Fit $_{3}$

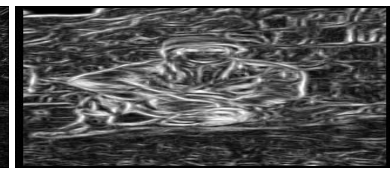

$T_{h g}$

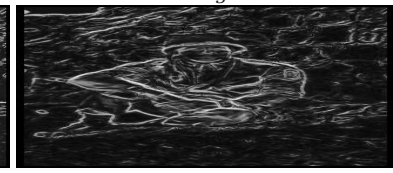

$\mathrm{Fit}_{30}$

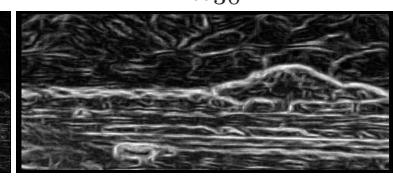

$T_{h g}$

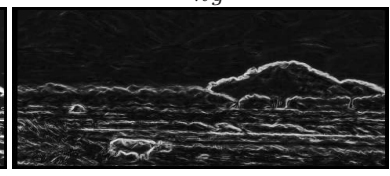

Fit $_{30}$

Fig. 7 Two example images detected by $T_{g g}, T_{h g}, T_{s d}, x_{b a y e s}$, and the best evolved programs from GP with $S e t_{b f, r a n d}$ using $\mathrm{Fit}_{3}$ and $\mathrm{Fit}_{30}$.

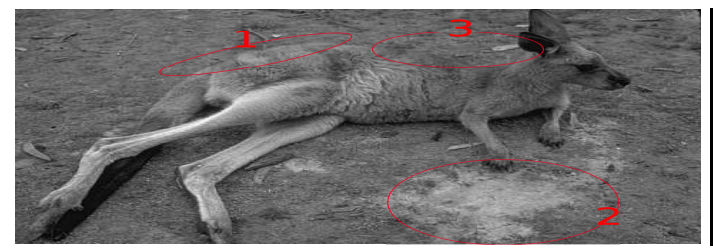

(a) Image

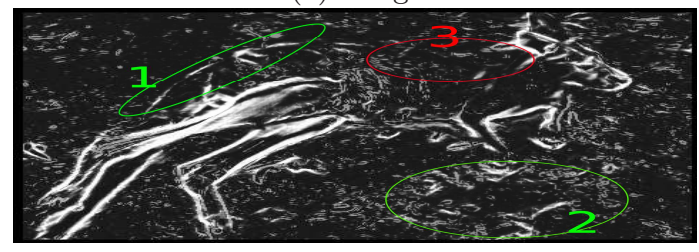

(c) GP

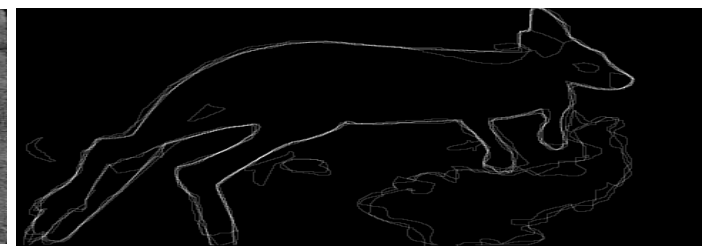

(b) Ground Truth

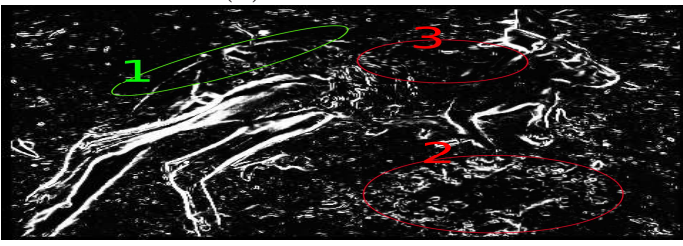

(d) $x_{\text {bayes }}$

Fig. 8 Image 69020 detected by an evolved program from Set $_{b f, \text { rand }}$ with Fit $_{3}$ and the combination $x_{\text {bayes }}$.

curves, it is found that the recall and precision values of the second part are almost located on the curve of $T_{h g}$. Different from $T_{h g}$, the recall and precision values of the second part of the evolved program crowd in an area (with not too low recall and not too low precision). From the view of the performance based on different thresholds, repeating estimation of a single variable with its estimated outputs transforms the variable values into a suitable range which is not strongly affected by the change of a threshold. Although neither the first part nor the second part is better than $T_{h g}$, the program combining the two parts is clearly significantly better.

\section{Further Discussions}

This section discusses the influence of the general algebraic operators, computational cost for the settings, and convergence of the GP system.

\subsection{Influence of General Algebraic Operators}

The results from Fit $_{3}$ and Fit 30 (in Tables 2 and 4 ) show that multiple thresholds obviously affect the test performance of the evolved programs. When Set $_{b t}$ and $\mathrm{Fit}_{3}$ are used, the GP system only finds $x_{\text {bayes }}$ as the final solution. However, when Set $t_{b t}$ and Fit $t_{30}$ are used, the evolved programs are significantly better the results from Set ${ }_{b t}$ with $\mathrm{Fit}_{3}$. 


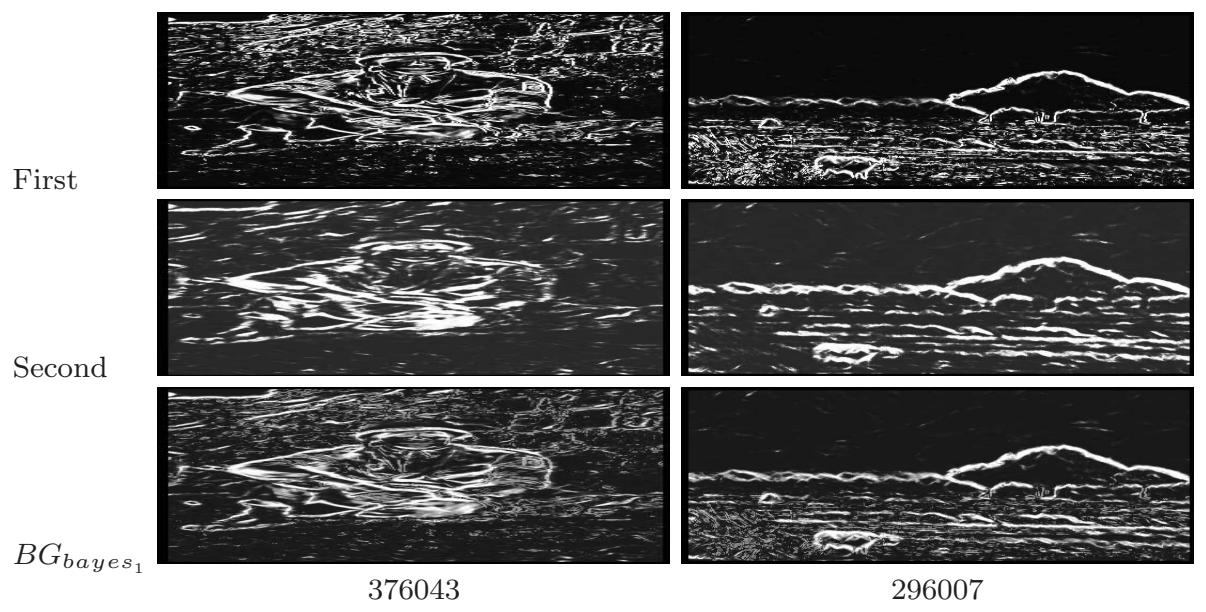

Fig. 9 Two example images detected by two parts of the best evolved method from GP.

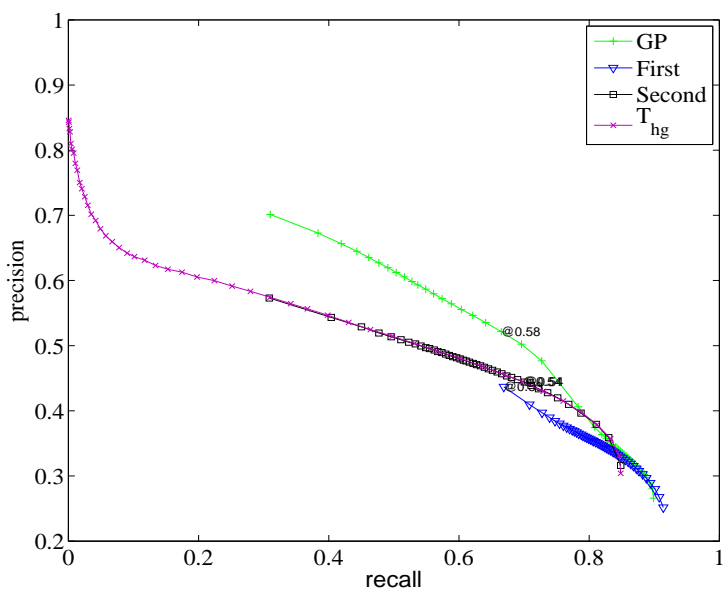

Fig. 10 Recall and precision for an evolved program, its two parts and $T_{h g}$.

Since $\mathrm{Fit}_{3}$ mainly focuses on selecting programs with high contrast edge responses, the two general algebraic operators do not work well on combinations of basic features. If the terminal set only includes $x$ and the function set only includes the two general algebraic operators, the test performance $\left(F_{\max }\right)$ for using $F_{i t}$ is $0.5459 \pm 0.0087$. From the evolved programs, it is found that 25 of the 30 evolved programs are equal to $T_{h g}$. Therefore, it is hard to find a good combination of basic features when $\mathrm{Fit}_{3}$ is used. This suggests that combining basic features with the two general algebraic operators might not be good to construct composite features with high contrast edge responses.

When $F_{i t} t_{30}$ is used to evolve programs, the terminal set only includes $x$, and the function set only includes the two general algebraic operators, the test performance $\left(F_{\max }\right)$ is $0.5714 \pm 0.0125$. As can be seen, only using the two operators can also obtain good com- posite features. The test performance on Set $_{b t}$ with $F_{i t}{ }_{30}$ is very close to the test performance without using $x_{\text {bayes. }}$. In order to check whether the evolved programs from Set $_{b t}$ with Fit $_{30}$ include any $x_{\text {bayes }}$, the evolved programs are simplified. From the simplification, the number of the evolved programs without $x_{\text {bayes }}$ is only nine out of 30 (less than one third). Therefore, $x_{\text {bayes }}$ is still helpful to construct good composite features. Equation (16) gives an evolved program $B G_{\text {bayes }_{2}}\left(F_{\max }=0.5941\right)$ from $S_{e t} t_{b t}$ with $F i t_{30}$, where $\frac{3}{8}$ and $\frac{5}{8}$ are scale parameters after simplification. The evolved program $B G_{\text {bayes } 2}$ includes $x_{\text {bayes }}, T_{h g}$ and $T_{g g}$.

$B G_{\text {bayes }_{2}}=\frac{3 T_{g g}}{8} \oplus \frac{5 T_{h g}}{8} \oplus\left[\left(T_{h g} \ominus T_{g g}\right) \ominus\left(x_{\text {bayes }} \oplus T_{h g}\right)\right]$

Fig. 11 shows two example images detected by $B G_{b a y e s}$, and an evolved program without $x_{\text {bayes }}$ (from Set bt $_{\text {us- }}$ ing $F i t_{30}$, and $\left.F_{\max }=0.5940\right)$. Comparing the visual results, the red circled areas (marked as number 1) in the two images detected by the evolved program without $x_{\text {bayes }}$ have lower edge responses than the relevant results from $B G_{\text {bayes }_{2}}$. A reason is that the responses on composite features combined by the general algebraic operators are dependent on the responses in the basic features, but Bayesian programs increase the edge response contrast. Since the technique only using general algebraic operators is different from the Bayesian technique, further investigation on both techniques in the GP system will be conducted in the future.

\subsection{Computational Cost}

Table 5 gives the training time (mean \pm standard deviations in seconds) for evolving programs by using the six settings respectively. Here, the $p$-value is obtained from 


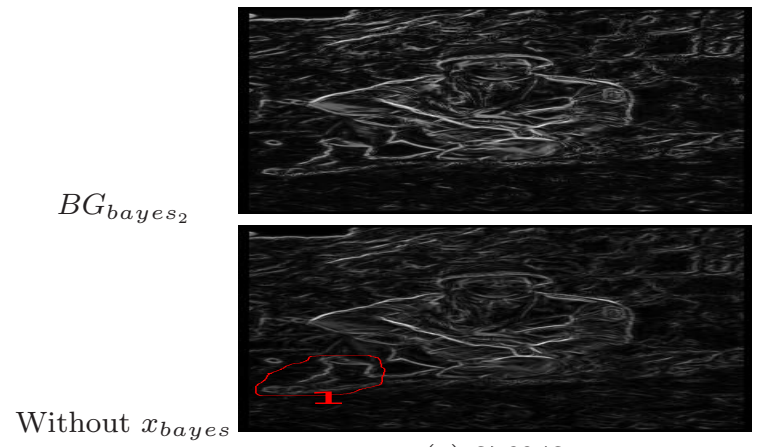

(a) 376043

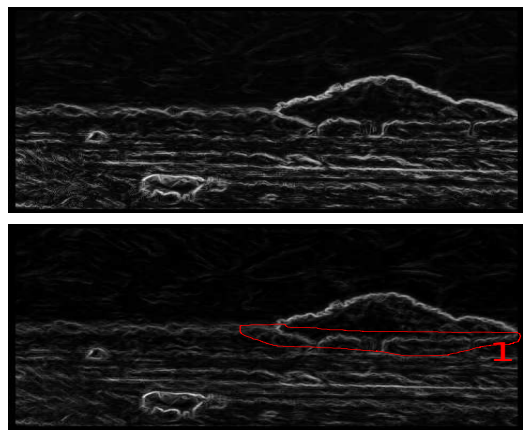

(b) 296007

Fig. 11 Two example images detected by two evolved programs from Set $_{b t}$ using Fit $t_{30}$.

Table 5 Training time (means \pm standard deviations in seconds) from $F_{i t}$ and $F i t_{30}$ using the six settings.

\begin{tabular}{c|ccc}
\hline Setting & Fit $_{3}$ & Fit $_{30}$ & $p$-value \\
\hline Set $_{b f, \text { rand }}$ & $190.9207 \pm 61.6907$ & $19.8340 \pm 13.6920$ & $0.0000 \downarrow$ \\
Set $_{b, \text { all }}$ & $73.6683 \pm 10.6519$ & $14.9423 \pm 12.3262$ & $0.0000 \downarrow$ \\
Set $_{b t}$ & $47.2473 \pm 11.3341$ & $73.2913 \pm 47.6183$ & $0.0131 \uparrow$ \\
Set $_{b t, \text { all }}$ & $314.3730 \pm 74.1631$ & $53.6197 \pm 46.0516$ & $0.0000 \downarrow$ \\
Set $_{b t, \text { rand }}$ & $111.5797 \pm 16.8419$ & $63.4163 \pm 41.6157$ & $0.0000 \downarrow$ \\
Set $_{\text {full }}$ & $167.5110 \pm 38.1950$ & $43.1970 \pm 38.7859$ & $0.0000 \downarrow$ \\
\hline
\end{tabular}

the comparison between the results from $\mathrm{Fit}_{3}$ and $\mathrm{Fit}_{30}$ in each setting by using a paired-sample $t$-test because of the same initial population. Note that $\downarrow$ means that the training time of using $F_{i t}$ is significantly longer than the training time of using $F_{i t}$, and $\uparrow$ means that the training from the former is significantly shorter than the latter. All experiments are run on single machines with CPU 3.1 GHz at the full speed state (the initial CPU speed is $1.6 \mathrm{GHz}$ for the power saving feature). Note that the value for $F_{i t}$ or Fit 30 is calculated after only visiting the training data once, so the calculation cost on Fit 3 and Fit 30 is very close when evaluating the same program.

From an overall view, the training time for each setting is quite short. The test time for detecting an image can be ignored (several milliseconds) because all basic features are loaded into the memory before executing a program.

From the table, the training time of using Fit $_{3}$ is significantly longer than the training time of using Fit $_{30}$ in each setting, except for Set $_{b t}$. Since subtrees including the two general algebraic operators might be important to construct a good program, the number of Bayesian functions might be reduced in the evolved good programs from Fit $_{30}$. Note that an algebraic operator has less computational cost than a Bayesian function. Since a Bayesian function needs to calculate the sample means and standard deviations of its input, the training cost from $\mathrm{Fit}_{3}$ is higher than the training cost from $F_{i t}{ }_{30}$, except for Set $_{b t}$. Since the evolved programs from Set $t_{b t}$ only do not include Bayesian functions, the training time of using Fit $_{30}$ in Set $_{b t}$ is longer than the training time of using Fit $_{3}$ in Set $_{b t}$. Also there is another reason for using $F_{i t}$ being longer than using Fit $_{3}$ for Set $_{b t}$, that is, Fit F $_{3}$ in Set $_{b t}$ only finds $x_{\text {bayes }}$ as final solutions; but Fit ${ }_{30}$ in Set $_{b t}$ needs to find good subtrees constructed by the two general algebraic operators, which takes some computational cost.

\subsection{Convergence}

Since the population size and maximum generation are very small in the settings, it is worth investigating the convergence of this Bayesian GP system. Fig. 12 reveals that the average best fitness at each generation in the six settings when Fit or Fit $_{30}$ is used. From Fig. 12 (a), since Set $_{b t}$ only finds $x_{\text {bayes }}$ as the final solutions, the best fitness value stays horizontal. The other settings almost reach constant values after generation 40 when Fit $_{3}$ is used. For Fit 30 (see Fig. 12 (b)), all settings are approximately convergent to constant values after generation 30. Set $t_{b, \text { rand }}$ and Set $_{b f, a l l}$ increase a bit in fitness after generation 43. Therefore, Fig. 12 (a) and (b) indicate that the GP system is convergent or very closely convergent at generation 50 .

\subsection{Number of Thresholds in Fit}

The fitness function $\mathrm{Fit}_{3}$ has the evolved results with high contrast edge responses, and the evolved results 


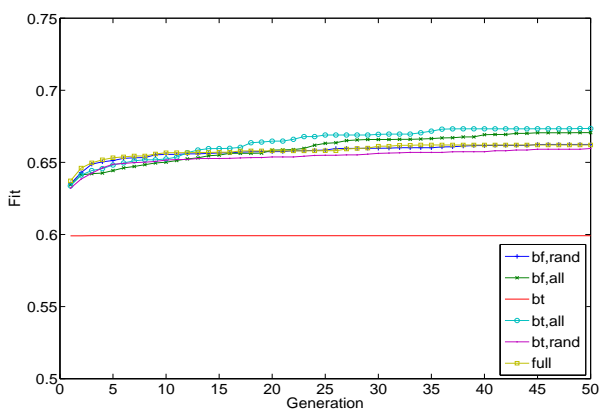

(a) Fit $_{3}$

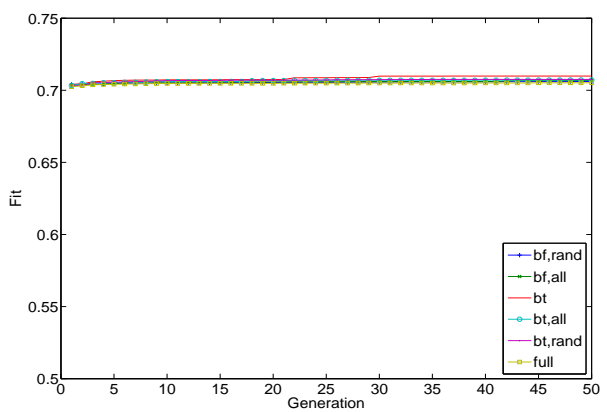

(b) Fit $_{30}$

Fig. 12 Means of the best fitness at each generation in each setting.

from Fit $_{30}$ decreases the responses on non-edge points which have strong responses in the results from $\mathrm{Fit}_{3}$. In order to investigate the influence of the number of thresholds in Fit, ten thresholds are used to evolve Bayesian programs when Set $_{b f, \text { rand }}$ is used. Here, we use $F_{i t}$ to indicate $F i t$ using ten thresholds. The test performance $F_{\max }$ for $F i t_{10}$ is $0.5763 \pm 0.0152$. The results from $F i t_{10}$ are significantly better the results from $\mathrm{Fit}_{3}$ and are not significantly different from the results from Fit $_{30}$.

Fig. 13 shows two example detected images from $\mathrm{Fit}_{3}, \mathrm{Fit}_{10}$ and $\mathrm{Fit}_{30}$. From an overview, there is no very obvious difference between the results from $F i t_{10}$ and $F_{i t} t_{30}$. Compared to the detected results from $\mathrm{Fit}_{3}$, the detected results from Fit $_{10}$ decrease the responses on some edges and discontinuities non-edge areas, such as the background of image 376043. Compared to the detected results from $F i t_{30}$, responses on some true edges in the results from Fit $_{10}$ are lower, such as the boundary of the tree in image 296007. It is possible that using a very small number of thresholds in Fit makes a good program with the observations of most non-edge points close to 0 and the observations of most edge points close to 1 .

When the number of thresholds used in Fit is neither small nor large, a good program can have the observations for edge points and non-edge points which are close but located in different ranges. Since the observations of programs are close and a range between two close thresholds is not very narrow, the edge response contrast for detected images from $F i t_{10}$ is lower than the contrast of the detected images from $\mathrm{Fit}_{3}$. When the number of thresholds used in Fit is large, each range between two close thresholds is narrow so that the observations of a good program for edge points and non-edge points are located in different ranges. It is possible that most distances from the observations of edge points to the observations of non-edge points in $F_{i t} t_{30}$ are longer than the relative distances in $F_{i t}$.
Therefore, the edge response contrast for the detected images from Fit $_{30}$ is higher than the relevant contrast from $F i t_{10}$. Note that the edge response contrast is a subjective evaluation, and it is not equal to the test performance $F_{\max }$. To obtain very high contrast edge responses, it is suggested that a small number of thresholds should be used in Fit. However, if weak responses on discontinuous non-edge areas are also considered, it is suggested that a large number of thresholds should be used in Fit.

\section{Conclusions}

The goal of this paper was to investigate automatic high-level feature construction for edge detection using GP and Bayes' theorem. The Bayesian technique was employed to combine basic features via using a general multivariate normal density. The goal was successfully achieved by evolving Bayesian programs with Bayesian functions and terminals, and two general algebraic operators.

From the results, firstly, the evolved Bayesian programs are better than the simple Bayesian model directly using the multivariate normal density, and also have high contrast edge responses. Secondly, when the fitness function only uses three thresholds to search programs with high contrast edge responses, the Bayesian function with randomly selected basic features is better than the function using the full set of basic features. However, when the fitness function uses 30 thresholds, there are no significant differences in terms of the test performance $F_{\max }$. Thirdly, in order to obtain better high contrast edge responses on composite features, using the general multivariate normal density as a function is better than using it as a terminal to evolve programs. Lastly, further analysis of the best evolved program reveals that iteratively estimating a single feature may help to improve detection performance. 


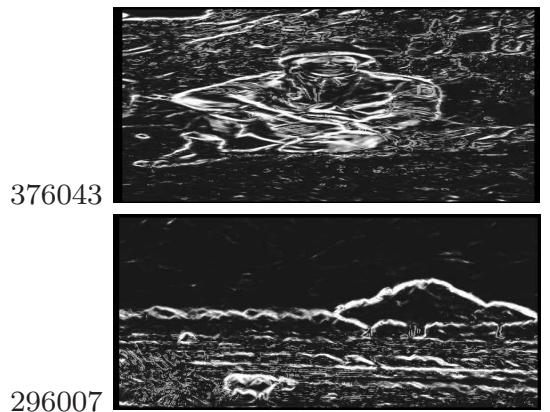

(a) $\mathrm{Fit}_{3}$

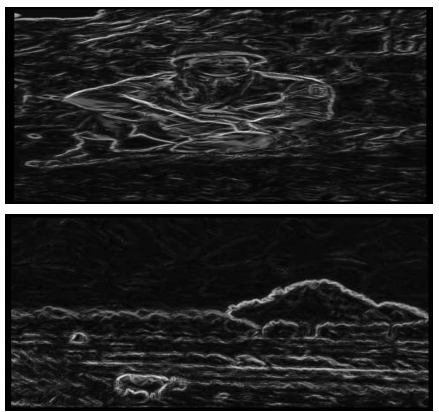

(b) Fit 10

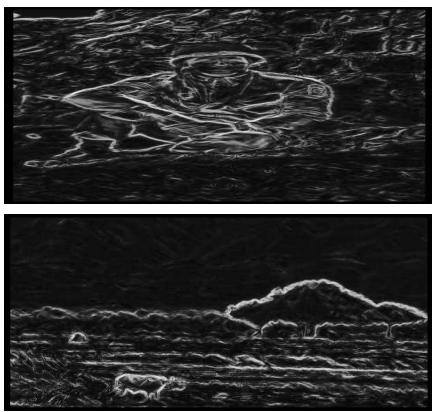

(c) Fit 30

Fig. 13 Comparisons among two detected example images from Fit $_{3}$, Fit $t_{10}$ and Fit $_{30}$.

For future work, we will investigate the influence of density functions used in multivariate distributions. Also, the Bayesian programs will be further manipulated for boundary detection.

\section{References}

1. Arbeláez P, Maire M, Fowlkes C, Malik J (2011) Contour detection and hierarchical image segmentation. IEEE Transactions on Pattern Analysis and Machine Intelligence 33(5):898-916

2. Azad P, Gockel T, Dillmann R (2008) Computer Vision: Principles and Practice. Elektor

3. Basu M (2002) Gaussian-based edge-detection methods: a survey. IEEE Transactions on Systems, Man, and Cybernetics, Part C: Applications and Reviews 32(3):252-260

4. Bolis E, Zerbi C, Collet P, Louchet J, Lutton E (2001) A GP artificial ant for image processing: preliminary experiments with EASEA. In: Proceedings of the 4th European Conference on Genetic Programming, pp 246-255

5. Canny J (1986) A computational approach to edge detection. IEEE Transactions on Pattern Analysis and Machine Intelligence 8(6):679-698

6. Dollar P, Tu Z, Belongie S (2006) Supervised learning of edges and object boundaries. In: Proceedings of the IEEE Computer Society Conference on Computer Vision and Pattern Recognition, vol 2, pp 1964-1971

7. Duda RO, Hart PE, Stork DG (2000) Pattern Classification (2nd Edition). Wiley-Interscience

8. Ebner M (1997) On the edge detectors for robot vision using genetic programming. In: Proceedings of Horst-Michael Gro $\beta$, Workshop SOAVE 97 - Selbstorganisation von Adaptivem Verhalten, pp 127134

9. Fernández-García N, Carmona-Poyato A, MedinaCarnicer R, Madrid-Cuevas F (2008) Automatic generation of consensus ground truth for the comparison of edge detection techniques. Image and Vision Computing 26(4):496-511

10. Fu W, Johnston M, Zhang M (2011) Genetic programming for edge detection: a global approach. In: Proceedings of the IEEE Congress on Evolutionary Computation, pp 254-261

11. Fu W, Johnston M, Zhang M (2012) Automatic construction of invariant features using genetic programming for edge detection. In: Proceedings of the Australasian Joint Conference on Artificial Intelligence, pp 144-155

12. Fu W, Johnston M, Zhang M (2012) Genetic programming for edge detection using blocks to extract features. In: Proceedings of the Genetic and Evolutionary Computation Conference, pp 855-862

13. Fu W, Johnston M, Zhang M (2012) Soft edge maps from edge detectors evolved by genetic programming. In: Proceedings of the IEEE Congress on Evolutionary Computation, pp 24-31

14. Fu W, Johnston M, Zhang M (2013) Genetic programming for edge detection using multivariate density. In: Proceedings of the Genetic and Evolutionary Computation Conference, pp 917-924

15. Ganesan L, Bhattacharyya P (1997) Edge detection in untextured and textured images: a common computational framework. IEEE Transactions on Systems, Man, and Cybernetics, Part B: Cybernetics 27(5):823-834

16. Golonek T, Grzechca D, Rutkowski J (2006) Application of genetic programming to edge detector design. In: Proceedings of the International Symposium on Circuits and Systems, pp 4683-4686

17. Grigorescu C, Petkov N, Westenberg MA (2004) Contour and boundary detection improved by surround suppression of texture edges. Image and Vision Computing 22(8):609-622

18. Harding S, Banzhaf W (2008) Genetic programming on GPUs for image processing. International Journal of High Performance Systems Architecture 


\section{1(4):231-240}

19. Harris C, Buxton B (1996) Evolving edge detectors with genetic programming. In: Proceedings of the First Annual Conference on Genetic Programming, pp 309-314

20. Hollingworth G, Smith S, Tyrrell A (1999) Design of highly parallel edge detection nodes using evolutionary techniques. In: Proceedings of the Seventh Euromicro Workshop on Parallel and Distributed Processing, pp 35-42

21. Jiang L, Wang D, Cai Z, Yan X (2007) Survey of improving naive bayes for classification. In: Proceedings of the 3rd International Conference on Advanced Data Mining and Applications, pp 134-145

22. Kadar I, Ben-Shahar O, Sipper M (2009) Evolution of a local boundary detector for natural images via genetic programming and texture cues. In: Proceedings of the 11th Annual Conference on Genetic and Evolutionary Computation, pp 1887-1888

23. Kokare M, Biswas PK, Chatterji BN (2003) Edge based features for content based image retrieval. Pattern Recognition 36(11):2649-2661

24. Kokkinos I (2010) Boundary detection using Fmeasure-, filter- and feature- (F3) boost. In: Proceedings of the 11th European Conference on Computer Vision: Part II, pp 650-663

25. Kruskal WH (1957) Historical notes on the Wilcoxon unpaired two-sample test. Journal of the American Statistical Association 52(279):356-360

26. Lam L, Lee SW, Suen C (1992) Thinning methodologies-a comprehensive survey. IEEE Transactions on Pattern Analysis and Machine Intelligence 14(9):869-885

27. Martin D, Fowlkes C, Tal D, Malik J (2001) A database of human segmented natural images and its application to evaluating segmentation algorithms and measuring ecological statistics. In: Proceedings of the 8th International Conference on Computer Vision, vol 2, pp 416-423

28. Martin D, Fowlkes C, Malik J (2004) Learning to detect natural image boundaries using local brightness, color, and texture cues. IEEE Transactions on Pattern Analysis and Machine Intelligence 26(5):530-549

29. Moreno R, Puig D, Julia C, Garcia M (2009) A new methodology for evaluation of edge detectors. In: Proceedings of the 16th IEEE International Conference on Image Processing (ICIP), pp 2157-2160

30. Papari G, Petkov N (2011) Edge and line oriented contour detection: state of the art. Image and Vision Computing 29:79-103

31. Poli R (1996) Genetic programming for image analysis. In: Proceedings of the First Annual Conference on Genetic Programming, pp 363-368

32. Rezai-Rad G, Larijani HH (2007) A new investigation on edge detection filters operation for feature extraction under histogram equalization effect. In: Proceedings of the Geometric Modelling and Imaging, pp 149-153

33. Shyi-Chyi C, Wen-Hsiang T (1994) Image compression by moment-preserving edge detection. Pattern Recognition 27(11):1439-1449

34. Song W, Feng G, Tiecheng L (2006) Evaluating edge detection through boundary detection. EURASIP Journal on Applied Signal Processing 2006:1-15

35. Sullivan J, Carlsson S (2002) Recognizing and tracking human action. In: Proceedings of the 7th European Conference on Computer Vision-Part I, pp 629-644

36. Wang J, Tan Y (2010) A novel genetic programming based morphological image analysis algorithm. In: Proceedings of the 12th Annual Conference on Genetic and Evolutionary Computation, pp 979-980

37. Zhang M, Cagnoni S, Olague G (2009) Gecco 2009 tutorial: Evolutionary computer vision. In: Proceedings of the 11th Annual Conference Companion on Genetic and Evolutionary Computation Conference, pp 3355-3380

38. Zhang Y, Rockett PI (2005) Evolving optimal feature extraction using multi-objective genetic programming: a methodology and preliminary study on edge detection. In: Proceedings of the Genetic and Evolutionary Computation Conference, pp 795-802 